\title{
Visualization of pilot flame of an optically-accessible coaxially- staged aero-engine lean-burn fuel injector
}

\author{
Kazuaki Matsuura*1, Shunya Uesaka², Tomoyuki Iwasaki², Yoji Kurosawa1, Hideshi Yamada', \\ Takeshi Yamamoto ${ }^{1}$ and Shigeru Hayashi ${ }^{2}$ \\ ${ }^{1}$ Institute of Aeronautical Technology, Japan Aerospace Exploration Agency, Japan \\ ${ }^{2}$ Department of Mechanical Engineering, Hosei University, Japan \\ ${ }^{*}$ Corresponding author: matsuura.kazuaki@jaxa.jp
}

\begin{abstract}
The visualization of the pilot flame of a coaxially-staged aero-engine lean-burn fuel injector, not only downstream but also inside of the pilot nozzle, was successfully performed at realistic aero-engine conditions. Optical access toward the inside of the nozzle was achieved through the inner and outer shrouds, both of which were made of transparent quartz. The image distortion caused by complex contours of the two shrouds was corrected by a method based on optical ray tracing, which realized precise determination of spatial intensity distribution of optical signals. Line-of-sight $\mathrm{OH}$ chemiluminescence, cross-sectional OH-LIF, kerosene LIF and kerosene Mie scattering were employed as diagnostic tools. The effects of pilot local air-to-fuel ratio on spray flame structure were revealed, both inside and downstream of the pilot nozzle under stable combustions. As the pilot mixture got rich, the main reaction zone moved from inside of the pilot nozzle to the region near the injector lip downstream of the injector exit. The OH-LIF signal was detected near the central axis surrounded by the fuel spray. It was also observed near the back-step of the pilot nozzle for the rich cases. The experiments under combustion oscillation were also conducted and the correlation of phenomena inside and downstream of the pilot nozzle was captured. It was clarified that the reaction enhancement in the outer part of the lip vortex region was caused by the convection of rich mixture, which appeared near the pilot atomizer lip at 150 210deg earlier oscillation phase angle.
\end{abstract}

\section{Keywords}

Aero-engine combustor, Optical burner/injector, Staged lean-burn injector, Laser induced fluorescence, Ray tracing, Optical refraction correction, Elevated temperature and pressure

\section{Introduction}

For reduction of NOx from aircrafts, coaxial-staging lean burn fuel injection systems have been studied intensively these days [1-15] and some of them have been already in service. Typically, they consist of a non-premixedmode pilot nozzle at the center for flame stability and a lean premixed-mode main nozzle surrounding the pilot for drastic NOx reductions. For such an injector, both fuel injection and fuel/air mixing start a few centimeter upstream from the injector exit (i.e. entrance of the combustion chamber). Moreover, this is also true for its pilot flame. Therefore, many important phenomena take place in regions enclosed by the metal walls of the injector, where the application of optical diagnostics is not straightforward compared with a conventional combustor.

In our previous work, visualization of fuel spray/vapor behavior and fuel/air mixing inside the main nozzle (i.e. the premixing duct) was performed by high speed visualization and/or LIF techniques $[10,11,15]$. Through a fullycontoured quartz-made transparent outer-shroud, dynamic motion of fuel sprays under combustion oscillations at an elevated pressure and temperature was clearly captured and discussed in relation to the flame dynamics downstream [15]. However, visualization of phenomena in the pilot nozzle has not been attempted yet.

In this work, the inner shroud of the staged injector, which was an annular metal block radially separating the pilot nozzle form the main, was also made of quartz. Therefore, the investigation of the pilot flame of the coaxial staged injector was made possible through both the inner and outer shrouds at realistic aero-engine conditions, which had been hardly found in published literatures to the authors' best knowledge. The present paper provides discussions on structures of spray flames both inside and downstream of the pilot nozzle at stable combustion conditions. In addition, the results on the correlation of the phenomena between these two regions under combustion oscillation are presented.

\section{Experiments}

Schematic drawings of the fuel injector are presented in Fig.1. Detailed descriptions of the coaxial-staging fuel injector can be found in Refs. [8,9]. The design of the pilot nozzle is briefly mentioned here for convenience of the discussions below. Two types of counter-rotating double-swirl airblast atomizers (for example, see Ref. [17]) were 
used, designated as U4D4 and U8. As for U4D4 type, liquid fuel (kerosene) is injected through four injection holes upstream and an annular exit at its atomizer lip. The liquid injected through the latter fuel circuit was metered by four grooves upstream of the exit to make annular liquid film as uniform as possible. The axes of the holes and grooves are inclined so that the liquid fuel is injected with swirling motions. As for U8, only upstream injection was employed through eight injection grooves and no injection at its atomizer lip. The U4D4 type is the standard design in our research project, whereas the U8 type was one of the prototype during the development. As mentioned later, the latter was used for study of combustion oscillation.
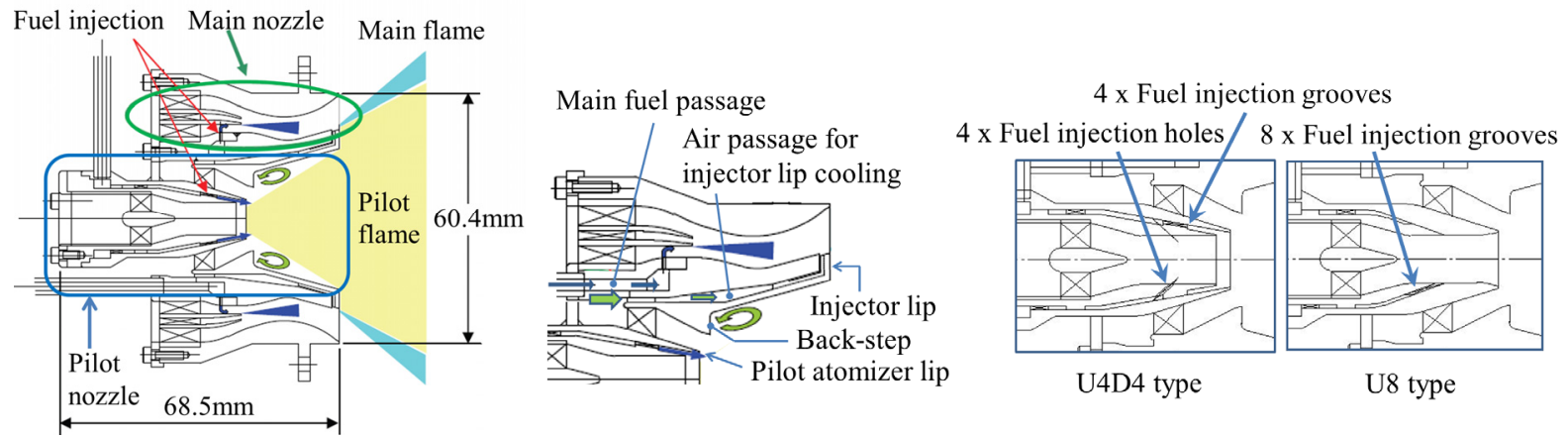

Figure 1. Staged fuel injector (Note the figure is for metal version with injector lip cooling).

The experimental setup is presented in Fig.2, which is similar to that used in our previous experiments $[11,15]$. The coordinate system used in this study is also shown in the figure, whose origin corresponds to the center of the exit of the fuel injector. For optical measurements, three optical accesses from top and sides were provided through quartz windows installed on the walls of the combustor liner and outer casing. The optical combustor has a square cross-section $(85 \times 85 \mathrm{~mm})$. For dynamic pressure measurements, a pressure transducer (Vibrometer CP211) was mounted on the sensor port on the lower wall of the combustor with a $28 \mathrm{~mm}$ recess. This enables introduction of cooling air for the sensor surface and also avoids its direct exposure to the flame.

To realize optical access toward the inside of the pilot nozzle, some modification was made from the original injector design. In addition to its outer shroud [15], its inner shroud was also partially made of transparent quartz. An air passage for the injector lip cooling and the main fuel passage in Fig. 1 were eliminated for simplification. Though this optical injector was originally designed to visualize the region of $z=-20.8 \sim-6.5 \mathrm{~mm}$, it was actually possible to observe phenomena close to the atomizer lip $(z \sim-22.8 \mathrm{~mm})$, owing to refraction effects. The region of $-6.5<z<1.5 \mathrm{~mm}$ is not visible because of the metallic heat shield.

The setup for visualization is shown in Fig.3, similar to Refs. [10-12,15] where its detailed descriptions are found. The laser sheet was parallel to the $y z$ plane and introduced from the top. Its thickness at the test section was approximately $0.5 \mathrm{~mm}$. In this study, the location of the laser sheet was fixed at the central plane $(x=0)$. The imaging optics was also similar to those in the references, except the CCD for Imaging optics A (PCO pixelfly

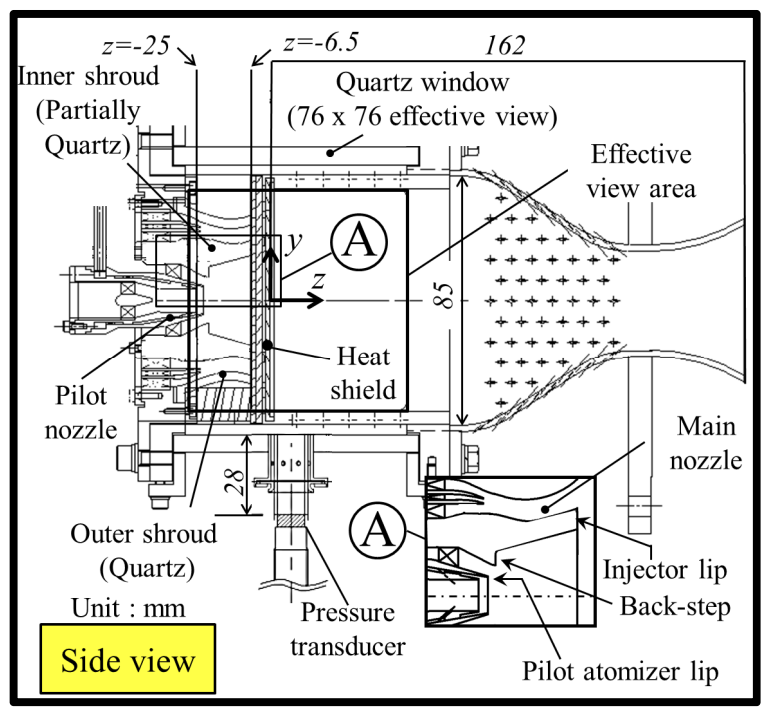

a) Optical combustor with optical injector.

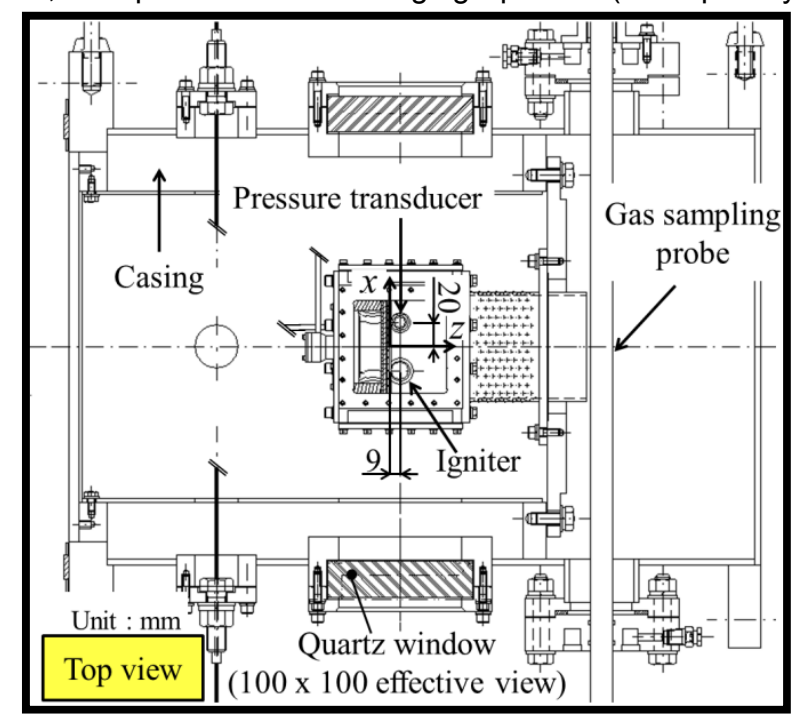

b) Combustor installed in test rig.

Figure 2. Schematic drawing of experimental setup. 
USB, 14bit). Imaging optics A could take images at higher acquisition rate $(10 \mathrm{~Hz})$ than imaging optics $\mathrm{B}(0.83 \mathrm{~Hz})$, but its image quality was not as good as that of the latter because the matching between relay-lens optics and the CCD was not optimized. They were originally used for simultaneous imaging, but not for the present experiments. Instead, only either of them was used depending on whether the image quality or acquisition rate was important. Images of $\mathrm{OH}$ chemiluminescence $\left(\mathrm{OH}^{*}\right.$, qualitative marker of heat release, location of reaction zones), OH-LIF (reaction zones and following burnt gas), kerosene-LIF (distribution of fuel in both liquid and gas phase, here tracing naphthalene-like molecules with two fused benzene rings,) and kerosene-Mie scattering (liquid phase fuel distribution) were taken by using appropriate optical band-pass filters. Those for $\mathrm{OH}^{*}, \mathrm{OH}-\mathrm{LIF}$, kerosene-LIF and kerosene-Mie scattering were CVI F10-307.1-3-2.00 (307.1nm, FWHM 10nm), Asahi Spectra MZ0310 (310nm, FWHM 10nm), Edmund \#84-108 (340nm, FWHM 29nm) and Asahi-Spectra MZ0280 (280nm, FWHM 10nm), respectively. The images of $\mathrm{OH}^{*}$ were those of line-of-sight, whereas the rests were those on the cross-sectional plane $(x=0)$. The signals for the laser trigger and camera acquisition timing were stored with the pressure oscillation signal by a multi-channel data recorder (TEAC, GX-1) at $100 \mathrm{kHz}$ sampling rate.

Experiments were conducted for two cases, Case A and B, as described in Table 1. Only pilot fuel was injected in all of the present experiments. Case $\mathrm{A}$ was to investigate the effects of pilot (local) air-to-fuel ratio $\left(A F R_{p}\right)$ on flame structures at stable combustion conditions, and Case B was to study dynamic behavior of the spray flame under combustion oscillation. For case $\mathrm{A}$, the variations of $A F R_{p}$ was carried out by changing the pilot fuel flow rate $\left(m_{f, p}\right)$ at a fixed inlet air condition. Here, $A F R_{p}$ was calculated using the ratio of effective opening areas between the pilot and the combustor in total, 110 and $760 \mathrm{~mm}^{2}$, respectively. A $10 \mathrm{~mm}^{2}$ reduction of the effective opening area of the combustor from the value in Ref. [15] was due to the elimination of the injector lip cooling. The inlet pressure, temperature, and combustor pressure loss for Case A were simulating a cruise condition of a target engine for small aircrafts $[8,9]$. Those of case B were close to its idle condition, but the inlet temperature was set about $40 \mathrm{~K}$ higher than the target value. This condition was a "trade-off" choice: The higher inlet temperature was, the better the image quality was, due to the less soot deposition to the transparent inner shroud, but the smaller amplitude of oscillation pressure it resulted in.

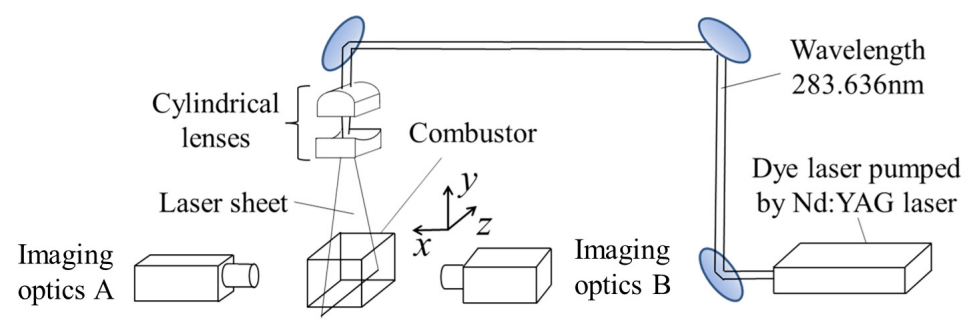

Figure 3. Optical setup.

Table 1 Test conditions.

\begin{tabular}{l|cc}
\hline \hline & Case A (Stable combustion) & Case B (Combustion oscillation) \\
\hline \hline Pilot nozzle type & U4D4 & U8 \\
Inlet pressure, $\mathrm{kPa}$ & 700 & 487 \\
Inlet temperature, $\mathrm{K}$ & 760 & 545 \\
Combustor pressure loss & $4 \%$ & $4 \%$ \\
Airflow rate in total $, m_{a}, \mathrm{~g} / \mathrm{s}$ & 316 & 259 \\
Pilot fuel flow rate, $m_{f, p}, \mathrm{~g} / \mathrm{s}$ & $1.52-5.44$ & 1.58 \\
Pilot air-to-fuel ratio, $A F R_{p}$ & $30.1-8.4$ & 23.8 \\
Imaging optics & B & $\mathrm{A}$
\end{tabular}

${ }^{*}$ Percent of inlet pressure

\section{Results and discussions}

A typical photograph of the pilot flame of the present staged-injector is presented in Fig. 4. The structure of the upstream part of the pilot flame is successfully revealed by means of the "optical staged-injector" developed for this study. The flame shows a conical structure, since the airflow is detached from the back-step of the pilot nozzle as illustrated in Fig. 1.

Image distortion is one of the serious problems on the visualization of phenomena inside the pilot nozzle. The complex contours of the inner and outer shrouds result in refractions of optical rays. To compensate this effect, a method was developed for optical refraction correction based on optical ray tracing. A pin-hole model was used to 


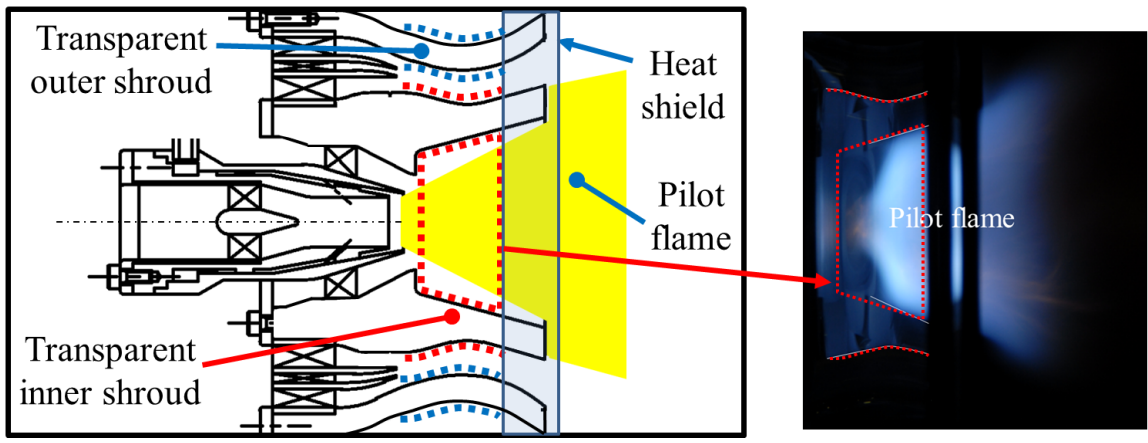

Figure 4. Visualization of flame in pilot nozzle and combustion chamber (Case A, $A F R_{p}=30.1$, Exposure time; 20ms).

model the imaging optics. The correction method is partially described in Ref. [16] with an example of its application to the visualization of fuel distribution inside the premixing duct (i.e. main nozzle) of the same injector. In this study, the same method was applied to the imaging of the pilot zone. The calculated degree of distortion in terms of displacements $(\Delta y, \Delta z)$ when imaging phenomena on a laser sheet at the central plane $(x=0)$ is presented in Fig. 5-a. Here, $\Delta y, \Delta z$ are defined so that the refraction correction was achieved by the transform of intensity distribution $I(y, z)$ to $I(y+\Delta y, z+\Delta z)$. The displacements were not calculated for regions without interests of this study, as masked by grey color. The importance of the correction is clear especially close to the pilot atomizer lip where the displacements are significantly large $(\Delta z>3.5 \mathrm{~mm})$. An original image for kerosene-LIF and that corrected by the present method are shown in Fig. 5-b. After the correction, the intensity distribution becomes realistic; for example, the upstream part of the fuel distribution attaches the pilot atomizer lip. The example shows that precise determination of spatial distribution of LIF signals can be realized by means of the present method.

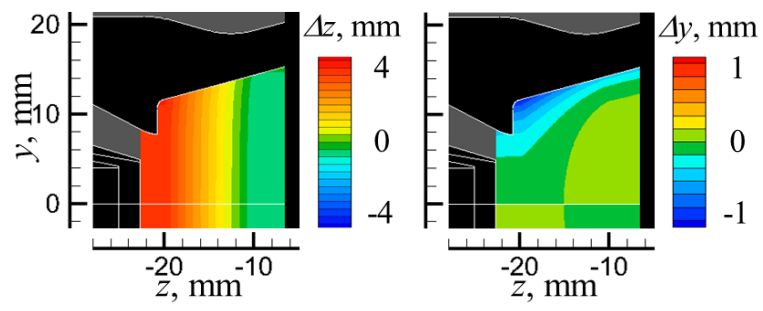

(a) Displacement caused by refraction
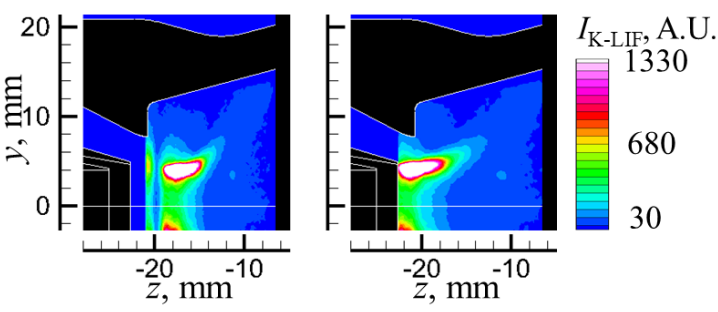

(b) kerosene-LIF intensity before(left) and after(right) correction

Figure 5. Optical refraction correction.

As mentioned in the following discussions, the interpretation of the results obtained from the present optical measurements is not always straightforward. Some items which should be taken into account for correct data interpretation are summarized in advance, in Table 2.

Spatial distribution of time-averaged intensity of line-of-sight $\mathrm{OH}$ chemiluminescence $\left(\mathrm{OH}^{*}\right.$-LoS), cross-sectional $\mathrm{OH}$-LIF, kerosene LIF and kerosene Mie scattering are presented in Fig.6 for Case A. Results for roughly upper half of the combustor $(y>0)$ are presented because the phenomena were nearly symmetric with respect to $z$ axis in a qualitative sense. The regions where the cautions are needed for data interpretation are indicated with the item numbers in Table 2. The structures of spray combustion field for two cases, a lean and a slightly rich ones $\left(A F R_{p}\right.$ $=25.1$ and 12.6) are summarized in schematic illustrations in Fig. 7. For convenience of explanation, reaction zones are separately numbered, (i) near the central axis and the atomizer lip close to the liquid spray, (ii) from middle and downstream part of the pilot nozzle extending to the injector lip region, (iii) close to the injector lip, (iv) from the injector lip extending to central region in the combustion chamber reflecting the inner recirculation zone (IRZ) flow structure, and (v) near the back-step.

For the leaner cases, the reactions in zones (i) (iii) are dominant, and they are in connection as seen in Fig. 6 $\left(\mathrm{OH}^{*}\right.$ and $\left.\mathrm{OH}-\mathrm{LIF}\right)$. OH-LIF signal is observed near the central axis surrounded by the fuel spray (designated as "A"). As $A F R_{p}$ decreases, zones (i) and (ii) look separated evidently ("B"), meaning the reaction is suppressed between the two. In this region, rich mixtures after evaporation exist, where the kerosene LIF signal is observed but the kerosene Mie is not. Thus, the separation is due to quick evaporation causing local temperature decrease due to its latent heat and too rich mixture, and both cause local suppression of reactions. On the other hand, reactions in the downstream part of zone (ii), and in zone (iii) are enhanced and still continue into zone (iv), as favorable mixture for combustion is established more downstream for low $A F R_{p}$ cases. For $A F R_{p}=12.6$, the 
Table 2. Items to be considered for data interpretation.

\begin{tabular}{|c|c|c|c|}
\hline Item No & Description & Location & Type of image \\
\hline 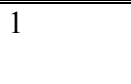 & Noises due to reflection and stray light & Close to walls and back-step & $\begin{array}{l}\text { All, especially } \\
\text { kerosene LIF }\end{array}$ \\
\hline 2 & $\begin{array}{l}\text { Noises due to reflection and stray light: } \\
\text { Correlated with signal intensity in combustion } \\
\text { chamber close to injector lip }\end{array}$ & $\begin{array}{c}\text { Downstream part of pilot } \\
\text { nozzle }\end{array}$ & OH LIF \\
\hline 3 & Insufficient cut-off by optical band-pass filter & $\begin{array}{l}\text { Near pilot atomizer lip where } \\
\text { kerosene is in liquid phase }\end{array}$ & $\mathrm{OH}$ LIF \\
\hline 4 & Transparency loss due to soot deposition & $\begin{array}{l}\text { Inside pilot nozzle, possibly } \\
\text { low } A F R_{p} \text { for Case } \mathrm{A} \text {, and } \\
\text { Case } \mathrm{B}^{* 1}\end{array}$ & All \\
\hline 5 & $\begin{array}{l}\text { Validity of correction of } \mathrm{OH} \text { chemiluminescence } \\
\text { line-of-sight image by applying displacement } \\
\text { values at the central plane }{ }^{\star 2}\end{array}$ & Inside pilot nozzle & $\begin{array}{l}\text { OH chemi- } \\
\text { luminescence }\end{array}$ \\
\hline
\end{tabular}

${ }^{*}$ For Case A, no soot deposition was observed for $A F R_{p}>18$ by visual inspection during the experiments. For $A F R_{p}<18$, direct observation was not reliable because of too bright background due to luminous flames so it was difficult to tell if it affects the signal quality. It should be noted that, at least, at $A F R_{p}=12.6$ for kerosene LIF experiment, its effect on the image is hardly observed. For Case $B$, the effect was apparent and must be considered for all the images.

${ }^{*}$ Present correction method should not be applied to line-of-sight images theoretically, but was still applied as the corrected images gave better qualitative information than the original images in practice.

kerosene vapor signal is detected even downstream of the injector exit ("C"). The $\mathrm{OH}^{*}$ signal overlaps it and the strong OH-LIF signal is observed its outside, behind the injector lip. This structure is caused by the lip vortex and some of the burned gas is trapped in this region. The existence of the lip recirculation zone (LRZ, [2]) was confirmed by our previous PIV measurements [13,14]. For further low $A F R_{p}$ cases (not shown), the location with OH-LIF signals in zone (iii) further moves towards outside with decreasing its intensity, as the mixture downstream of the injector becomes too rich. As for zone (v), the OH-LIF signal begins to be evident for $A F R_{p}=12.6$. This is mainly from that of burned gas. For rich cases such as for $A F R_{p}=8.4, \mathrm{OH}^{*}$ as a sign of heat release is detected ("D"), though its intensity is not strong.

The results for Case $\mathrm{B}$ are discussed in the followings. A typical pressure oscillation signal and its frequency spectrum after an adequate band-pass frequency filtering $(200-2500 \mathrm{~Hz})$ are shown in Fig. 8 . The number of FFT points was 8192 . The filtering was performed to remove low frequency noises mainly from the power supply line of the electric heater and its harmonics (especially 50 and $150 \mathrm{~Hz}$ ), and high frequency noises due to the acoustic resonance inside the pressure sensor mount port $(2800 \sim 4200 \mathrm{~Hz})$. The characteristic frequency of pressure oscillation is $390 \mathrm{~Hz}( \pm 8 \%)$.

The phase-averaged kerosene/OH LIF intensity distributions on $x=0$ plane are shown in Fig. 9 by line-contours and color plots, respectively. Here, the phase angle $\varphi$ is defined assuming that the pressure oscillation signals are in a sinusoidal shape, i.e., $p ; \sin (\varphi)$. The regions where the cautions are needed are again indicated with the item numbers. Even after the trade off choice of the test condition, the soot deposition (item 4 in Table 2) was still inevitable. Nevertheless, qualitative and semi-quantitative assessments were possible, providing information such as the correlation of phenomena inside and downstream of the pilot nozzle as described below: The phasedependent variations of the sum of LIF signals in some specified regions are presented in Fig. 10 as percentages of their average values. As shown in Figs. 9 and 10, the kerosene LIF intensity just downstream of the pilot atomizer lip (R1) shows its maximal value at $\varphi$ 330deg. This high concentration fuel parcel convects towards downstream at local flow speeds. Eventually, when assuming near-sinusoidal behaviour of fuel concentration variations and neglecting ripple-like structure of the curves in Fig. 10, the highest fuel concentration takes place at $\varphi \sim 0,60$, and $90 \mathrm{deg}$ for regions R2,R3 and R4 (z -13, -8 and 3mm), respectively. After the arrival of the high concentration fuel parcel, a reaction enhancement in the lip vortex region takes place. The rich mixture extends around the the lip vortex flow and encounters a flesh airflow from the main nozzle, which probably causes the reaction enhancement and apparent flash-back-like motion of the flame (designated as "A"). As a result, the $\mathrm{OH}$ LIF in the outer part of the lip vortex region (R5) shows its high values for $\varphi=120 \sim 180 \mathrm{deg}$, which is about 150 210deg later than the occurrence of the maximal fuel concentration in region R1. 

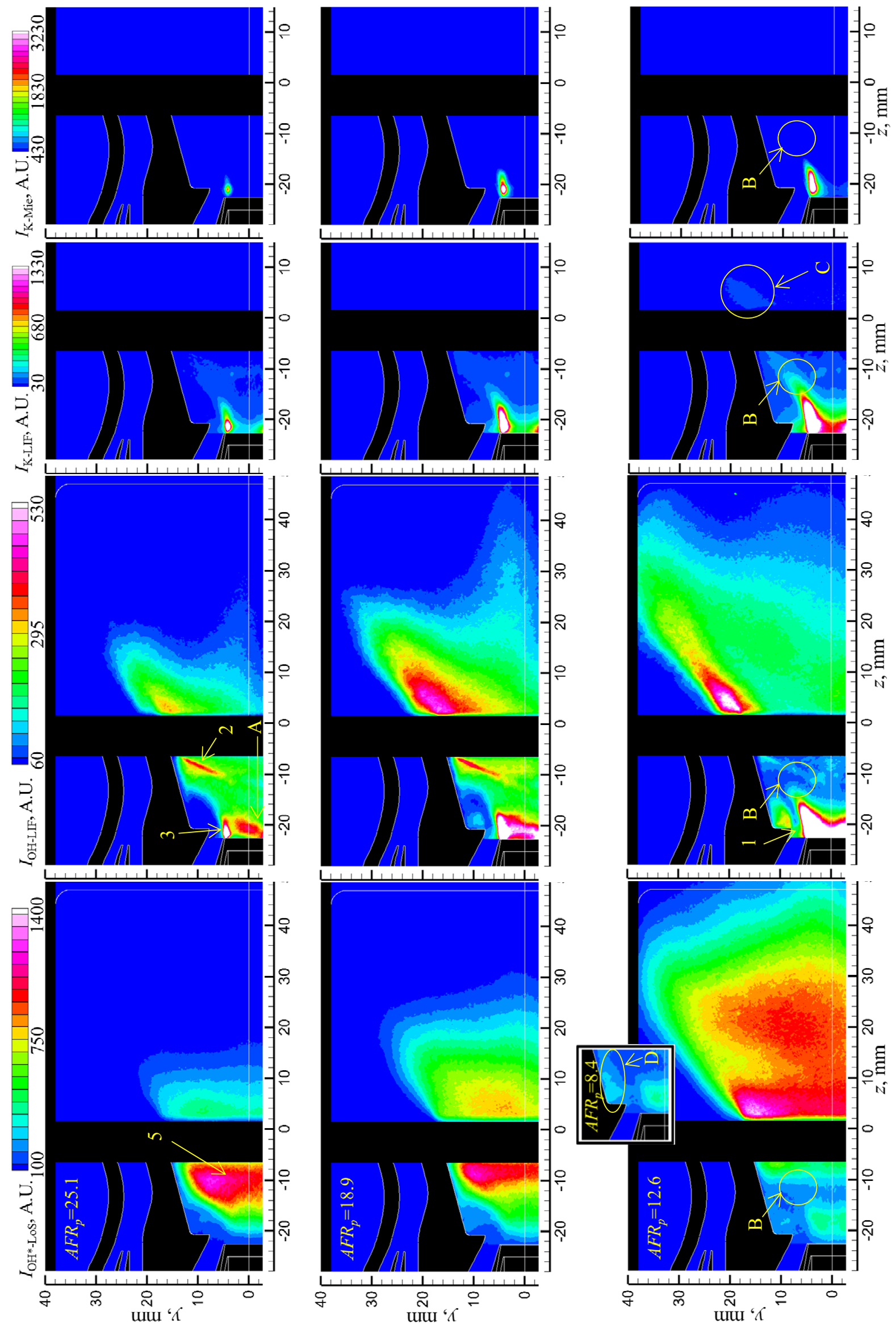

Figure 6. Spatial distribution of time-averaged intensity of line-of-sight $\mathrm{OH}$ chemiluminescence, cross-sectional $\mathrm{OH}-\mathrm{LIF}$, kerosene- LIF and kerosene Mie scattering on $x=0$ plane for Case $\mathrm{A}$ (note the figure is rotated).

This work is licensed under a Creative Commons 4.0 International License (CC BY-NC-ND 4.0).

EDITORIAL UNIVERSITAT POLITĖCNICA DE VALĖNCIA 


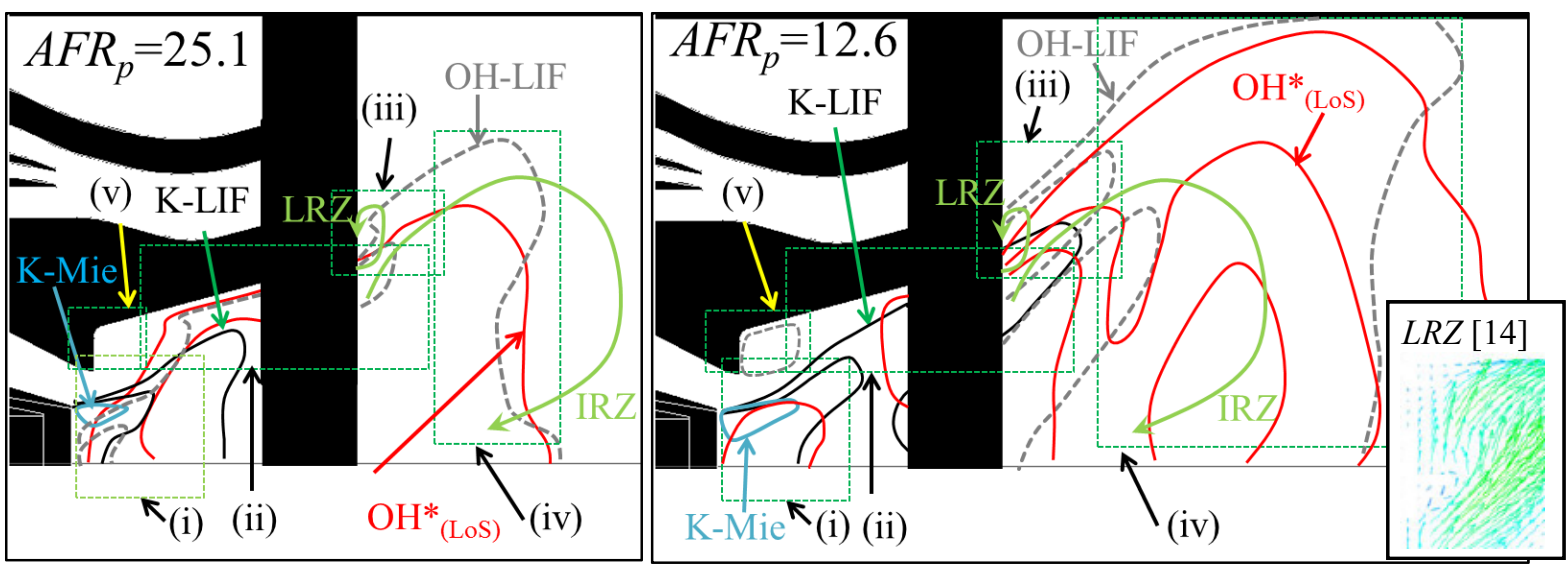

Figure 7. Schematic illustrations of pilot flame structure.
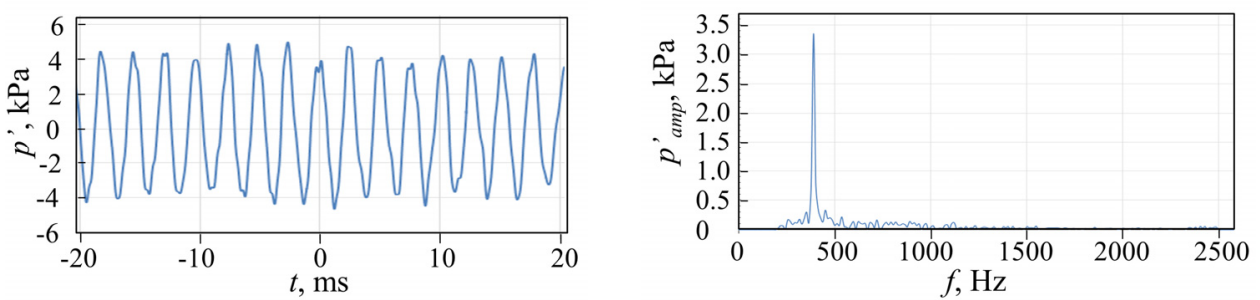

Figure 8. Examples of pressure oscillation signal and its frequency spectrum.

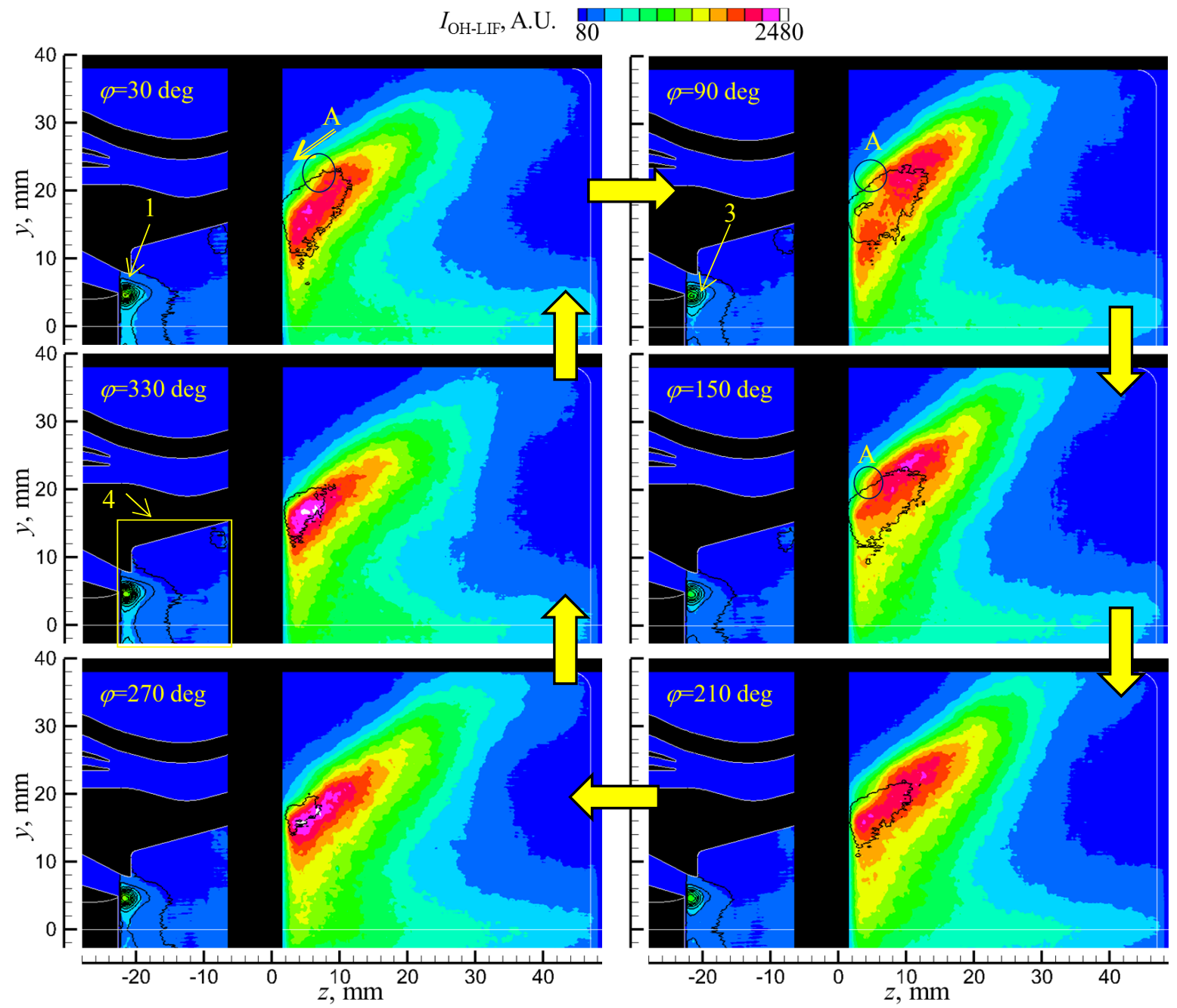

Figure 9. Phase-averaged kerosene/OH-LIF intensity distribution on $x=0$ plane for Case $\mathrm{B}$.

This work is licensed under a Creative Commons 4.0 International License (CC BY-NC-ND 4.0).

EDITORIAL UNIVERSITAT POLITĖCNICA DE VALÈNCIA 

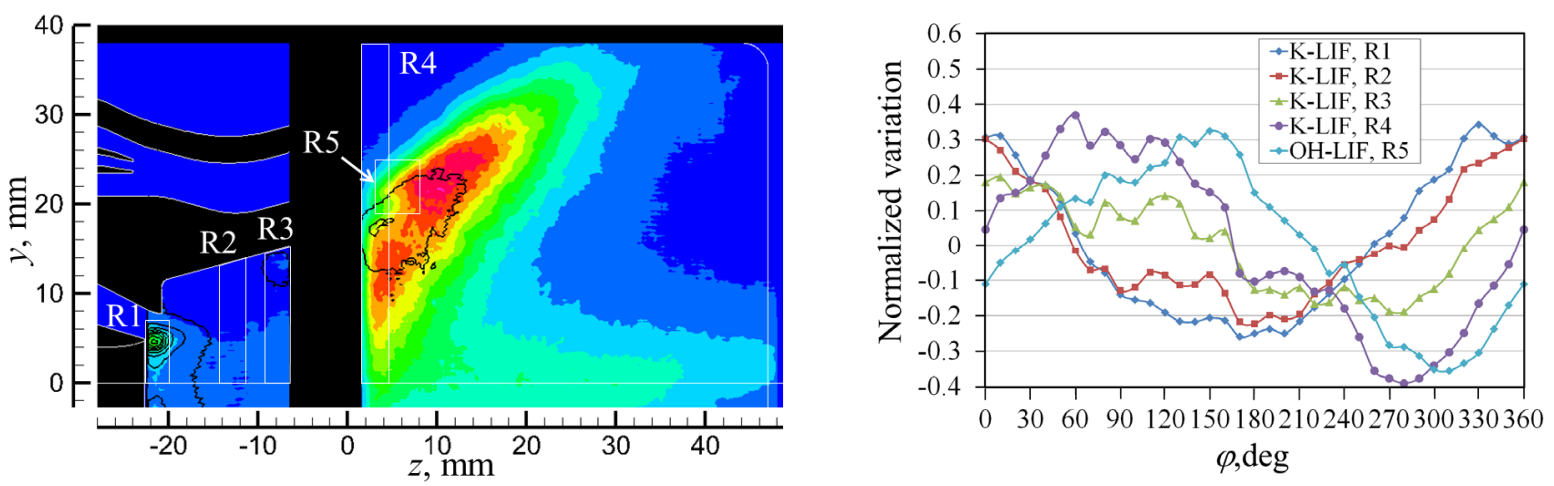

Figure 10. phase-dependent variation of the sum of LIF signals in specified regions.

\section{Conclusions}

The visualization of the pilot flame of the coaxially-staged aero-engine lean-burn fuel injector, not only downstream but also inside of the pilot nozzle, was successfully performed at the realistic aero-engine conditions. The optical access toward the inside of the nozzle was achieved through the inner and outer shrouds, both of which were made of transparent quartz. The image distortion caused by the complex contours of the two shrouds was corrected by the method based on the optical ray tracing. The line-of-sight $\mathrm{OH}$ chemiluminescence, crosssectional OH-LIF, kerosene LIF and kerosene Mie scattering were employed as diagnostic tools. The effects of the pilot local air-to-fuel ratio on the spray flame structure were revealed. As the pilot mixture got rich, the main reaction zone moved from inside of the pilot nozzle to the region near the injector lip downstream of the injector exit. The OH-LIF signal was detected near the central axis surrounded by the fuel spray. It was also observed near the back-step for the rich cases. The experiments under combustion oscillation were also conducted. The reaction enhancement in the outer part of the lip vortex region was observed as a result of the convection of rich mixture, which appeared near the pilot atomizer lip at 150 210deg earlier oscillation phase angle.

\section{References}

[1] Mongia, H.C., 2003, AIAA/ICAS International Air and Space Symposium and Exposition, AIAA 2003-2657. [2] Dhanuka, S. K., Temme, J. E., Driscoll, J. F., Mongia, H. C., 2009, Proc. Combustion Institute, 32, pp. 29012908.

[3] Lazik, W., Doerr, Th., Bake, S., v. d. Bank, R., Rackwiz, L., 2008, Proc. ASME Turbo Expo, GT2008-51115.

[4] Apeloig, J. M., d'Herbigny, F.X., Simon, F., Gajan, P., Orain, M., Roux, S., 2015. J. Prop. Power, 31(1), pp. 309-319.

[5] Fujiwara, H., Matsuura, K., Shimodaira, K., Hayashi, S., Kobayashi, M., Oda, T., Horikawa, A., Matsuyama, R., Ogata, H., Kinoshita, Y., 2011, Proc. ASME Turbo Expo, GT2011-46256.

[6] Matsuyama, R., Kobayashi, M., Ogata, H., Horikawa, A., Kinoshita, Y., Proc. ASME Turbo Expo, GT201268272.

[7] Yamamoto, T., Shimodaira, K., Kurosawa, Y., Matsuura, K., and Yoshida, S., 2009, Proc. ASME Turbo Expo, GT2009-59852.

[8] Yamamoto, T., Shimodaira, K., Yoshida, S., Kurosawa, Y., 2013, J. Eng. Gas Turbines Power, 135 (3), pp. 031502-031509.

[9] Yamamoto, T., Shimodaira, K., Kurosawa, Y., and Yoshida, S., 2013, Proc. ASME Turbo Expo, GT201395496.

[10] Matsuura, K., Ohori. S., Yoshiura, Y., Kurosawa, Y., Yamada, H., Shimodaira, K., and Hayashi, S., 2012, Poster presentation, 12th International Conference on Liquid Atomization and Spray Systems.

[11] Ohori. S., Eguchi, T., Matsuura, K., Yamada, H., Kurosawa, Y., Yamamoto, T., Shimodaira, K., Hayashi, S., 2013, Proc. 22th Japanese Conference on Liquid Atomization and Spray Systems, pp. 406-409 (in Japanese).

[12] Tachibana, S., Saito, K., Yamamoto, T., Makida, M., Kitano, T., Kurose, R., 2015, Combust. Flame, 162, pp. 2621-2637.

[13] Yoshiura, Y., Matsuura, K., Kurosawa, Y., Yamada, H., Shimodaira, K., Yamamoto, T., Hayashi, S., 2012, Proc. Gas Turbine Society of Japan, 40, pp. 159-164 (in Japanese).

[14] Matsuura, K., Ide, K., Yoshiura, Y., Ohori, S., Kurosawa, Y., Yamada, H., Shimodaira, K., Yamamoto, T., Ohota, Y., Hayashi, S., 2013, Proc. 25th European Conference on Liquid Atomization and Spray Systems, 2013.

[15] Matsuura, K., Eguchi, T., Oide, S., Yamada, H., Kurosawa,Y., Yamamoto, T., Hayashi, S., 2015, 51st AIAA/SAE/ASEE Joint Propulsion Conference, AIAA 2015-4085.

[16] Uesaka, S., Eguchi, T., Oide, S., Matsuura, K., Hayashi, S. 2016, Proc. 25th Japanese Conference on Liquid Atomization and Spray Systems, pp. 58-61 (in Japanese).

[17] Zheng, Q. P., Jasuja, A. K. Lefebvre, A. H., 1997, J. Eng. Gas Turbines Power, 119, pp. 512-518. 